\title{
Littérature et médecine: deux mondes incompatibles
}

\author{
Essai \\ C. Luezior
}

Dans un premier temps, essayez de vous mettre dans la peau d'un homme ou d'une femme souffrant d'une tumeur cérébrale accessible au scalpel. Franchement, aimeriez-vous être opéré par un neurochirurgienpoète? Sans hésitation: non, non, non, trois fois non. Le vocable poète étant pour la plupart de nos contemporains synonyme de rêverie, de flou artistique, de flirt avec des muses imprécises. Non, je ne ferai pas fendre mon crâne par un poète, fût-il chef de clinique ou professeur.

Que diriez-vous d'un pneumologue auscultant vos chères alvéoles alors que vous le savez écrivain? Malaise! Que va-t-il sussurer à propos de vos cavernes tuberculeuses? Va-t-il prendre sa lyre pour accompagner vos sibilances? Va-t-il faire son miel de vos insomnies alors que vous scandez vos quintes à tous vents? Franchement, le doute est insupportable.

Que diriez-vous d'un gynécologue déclamant une ode à Jules ou Alexandre, alors qu'un confrère dans la même situation hésiterait entre forceps et césarienne? Non, trois fois non!

Et l'eczéma de la Mère Michel va-t-il se retrouver dans une sombre intrigue policière où cellules et squames donneront finalement leurs empreintes génétiques pour signer le crime?

Il faudra bien la blouse blanche pour stériliser le fantasme et séparer l'ordonnance du poème.

Disons-le tout de go: un chirurgien vasculaire faisant partie d'une équipe universitaire sur la brêche vingt-quatre heures sur vingt-quatre est un homme probablement perdu pour la poésie. Ite missa est.

L'un de mes confrères psychiatres m'a toutefois confié la remarque suivante: «Tout le monde sait que les psy sont fous ou qu'ils ont choisi cette branche par courtoisie envers la folie. Cela, pour satisfaire l'inconscient collectif. En réalité, le fait que j'écrive des Krimis, en français polars", poursuit mon ami, «redonne du tonus et un pichet de confiance à mes patients. Quand le psy se transforme en Maigret, sa pipe retrouve une toute autre légitimité. Rechercher le coupable, c'est-à-dire poser un diagnostic différen-

Correspondance:

Claude Luezior

c/o C.-A. Dessibourg

Covy 30

CH-1754 Avry tiel dans la foule des symptômes a quelque chose de noble, de compréhensif, de louable. Le thérapeute un peu trop inspiré acquiert ainsi, de facto, l'aura du justicier. De celui qui cherche et qui trouve surtout."

Mais on ne peut évidemment pas tous être psychiatres écrivant des polars.

Nous concèderons bien entendu à l'interniste un certain professionnalisme dans la description de l'alcoolique et du toxicomane. Nous savons toutefois que la Faculté n'aida guère Verlaine ou Baudelaire. Les échecs ne se comptent plus: pensons à l'épilepsie supposée d'un St-Paul ou à celle d'un autre saint laïque, Van Gogh; méditons à propos de la syphilis inefficacement traitée d'un Daudet ou d'un Kafka. La maladie n'a-t-elle parfois joué un rôle créateur chez ces êtres exceptionnels, au même titre que la solitude, la fée bleue, l'incompréhension ou le désespoir? En rabotant la misère, n'eût-on pas lissé la création? La question reste ouverte ...

Relations difficiles, vous en conviendrez, avec ces médecins qui veulent stériliser, extirper, normaliser le terreau si infectieux mais si fertile de la nature humaine ...

Deux mondes incompatibles décidément, l'un se méfiant de l'autre. "Toi le neurologue, ne touche pas à mon pote poète avec tes neuroleptiques. Toi le généraliste, permet à Utrillo sa bouteille divine. Et toi, le psychothérapeute, laisse Cioran courir après son destin!»

Et puis, le secret médical réputé aussi inoxydable qu'un secret-défense, se charge bien de conserver pierre par pierre la muraille de Chine entre les deux professions. Et puis, la condescendance des deux mondes dans leurs tours d'ivoire respectives nous permet de vérifier l'imperméabilité de ces sociétés qui vivent côte à côte sous forme de classes, de castes, avec leurs hiérarchies, leurs valeurs, leurs réseaux. 0 tempora, o mores! N'avons-nous rien inventé?

Pourtant, que d'écrivains souffrant dans nos consultations, que d'alcôves ouvertes, que de plumes ulcérées, que d'inspirations à la dérive! Finalement, pourquoi le plumitif n'aurait-il pas droit à sa névrose, pourquoi le rimailleur n'aurait-il sa presbytie et l'essayiste, sa démence? Pourquoi nos frères en écriture n'auraient-il l'avantage de se réfugier comme tant d'êtres humains, dans le giron du colloque singulier? Plus que d'autres peut-être, ont-il soif d'une écoute attentive ou d'une poignée de main? Passerelle fragile d'Aspirines et d'antibiotiques, me direz-vous? A voir! 
La morbidité des poètes n'est en fait pas mon propos. Car vous n'attendez ici ni une consultation publique ni une leçon de statistiques. Car ce que j'aimerais vous dire au contraire, c'est ceci: je ne comprends pas pourquoi il n'y a pas davantage d'écrivains-médecins. Bien entendu, chacun a son expérience de vie, son transsibérien comme Cendrars, son bar à Cuba comme Hemingway, son avion à hélice comme St-Exupéry. Certains voyagent au bout de la terre à pied, à cheval ou en voiture, tel le poète, au gré de son imaginaire. D'autres ont besoin de combats tel Péguy, Sartre ou Malraux. Le médecin, lui, est au seuil privilégié de la naissance et de la mort. De l'amputation et du cri. Du grand brûlé qui tend ses doigts ou du misérable qui hurle dans sa psychose. Il palpe la crampe et le désarroi, la solitude qui tue à petite flamme et les fièvres qui font délirer l'être.

Cette pâte humaine a bien entendu inspiré nombre de témoignages plus ou moins réussis, d'auto-biographies dont l'essentiel fut de montrer la si belle valeur de l'homme en blanc! Elle est à la source de feuilletons télévisés pour mères de famille repassant leur linge avec indolence, mais assoiffées quelque part de frissons et d'urgences. Ces démarches, quelles soient commerciales ou ingénues ne sont bien sûr ni de l'art, ni du cinéma, ni de la littérature. Elles ont en fait un côté voyeuriste. Comme si Michel-Angepaparazzi eût simplement photographié, clic-clacKodak, la fuite du jardin d'Eden. Franchement: c'eût été indécent.

D'un côté, l'art lui-même par sa recherche d'absolu, l'écriture authentique par son désir d'adéquation totale à la vérité, ont eux, la légitimité nécessaire pour traduire la souffrance ou l'espoir de nos semblables. D'autre part, l'expérience de vie, qu'elle soit celle d'un coopérant au Bangladesh, d'un journaliste à Sarajevo, d'un infirmier au Ruanda, d'un chirurgien enlevant une cataracte au Mali ou d'un simple praticien dans son cabinet, peut être, par une démarche personnelle, à la source d'une inspiration forte.

La voilà, toute la dualité: art - expérience. Le voilà, le grand malentendu: écriture - vie. Tant d'hommes et tant de femmes sont dignes de laisser une trace ... Et pourtant, la plupart s'évaporeront sans en laisser aucune.

Par ailleurs, un certain nombre de plumitifs retournent leurs pages blanches à la recherche d'une pâle histoire à raconter et d'une muse anémique. Alors que le monde autour d'eux crève de cynisme, de meurtres et d'arrogance! Pas besoin d'être toubib pour déchiffrer les injustices et les guerres qui ne cessent de fleurir, la torture des camps et la soif du désert.

Ainsi, la voilà donc, la grande incongruité! Pourquoi le thérapeute, le praticien, le médecin aux pieds nus dans la glaise humaine n'ont-ils l'habileté de traduire leur expérience en expression artistique? Par manque d'inspiration peut-être? Parce que Calliope se méfie du serpent d'Esculape? Par manque de temps, disent-ils? La belle excuse; les apôtres eux aussi dormaient alors que le Christ était au jardin de Jetsemani.

Il y a bien sûr un Céline qui va au bout de la nuit avec son écriture d'écorché vif. Il y a Duhamel qui traduit avec passion ses actes de chirurgien dans la boue de la première guerre mondiale: lire la bouleversante Vie des martyrs à ce propos! Il y a Tchekhov qui n'a cessé de pratiquer la plume d'une main et le secours dérisoire de l'autre main. Il y a Rabelais, tout à la fois docte et charnel, il y a Conan Doyle avec ses descriptions minutieusement cliniques. Il y a Henri Mondor disséquant l'âme de Mallarmé et de Valéry, Starobinski le brillant critique, l'humaniste Jean Bernard et quelques autres. Finalement, bien peu de médecins-écrivains dans l'immense cohorte d'Hippocrate.

Alors bien sûr, quand un de ces disciples se présente sur l'île des poètes, on a tendance à l'éconduire, au nom de l'imaginaire. Au nom d'une dualité qui doit rester double, impénétrablement double. D'un côté l'action et de l'autre la mise en mots. On aurait pu dire d'un côté le glaive, le scalpel et de l'autre le forgeron, le barde. Etre les deux à la fois est une gageure. Il est en ainsi depuis toujours.

Mais il en est certains que taraudent malgré tout le verbe et l'image, l'intime frottis des mots, les hachures de la ponctuation et la respiration des paragraphes.

Et si la nouvelle renaissance était celle du réseau? Et si nous prenions Monsieur Inter Net au mot? Et si notre monde était finalement une toile où les gens venaient à se parler entre professions, entre castes? Comme Martin Luther King, j'ai un rêve. Et ce rêve, c'est celui de l'interface et de mains immenses, tentaculaires. Serrées entre les deux mondes. 